\title{
A Temperature Independent Driver for Mach-Zehnder Modulators
}

\author{
Shenghao Liu, Dr. Ke Li, and Dr. Peter Wilson \\ Electronics Electrical Engineering Group, School of Electronics and Computer Science \\ University of Southampton, UK \\ $\{$ sl29g11, kl, prw\}@ soton.ac.uk
}

\begin{abstract}
This paper describes a fully differential inductorless temperature independent laser driver for a Mach-Zehnder modulator (MZM). The laser driver in this work exhibits robustness in bandwidth and gain across the temperature range $27^{\circ} \mathrm{C}$ to $135^{\circ} \mathrm{C}$. This driver (fabricated using a 130nm CMOS process) can achieve a differential output voltage swing of up to $4 V$ pp when driving a $50 \mathrm{Ohm}$ Load at speeds of up to $12.5 \mathrm{~Gb} / \mathrm{s}$ across a temperature range $27^{\circ} \mathrm{C}$ to $135^{\circ} \mathrm{C}$.
\end{abstract}

Index Terms-Laser driver, low power, low cost, temperature variation, optical modulator.

\section{INTRODUCTION}

Due to the speed limitations of traditional copper based communications networks, there has been a drive towards optical fibre based systems to improve data throughput with the integration of Silicon circuits with Photonic Systems leading to the new field of Silicon-Photonics. It is likely that shortrun communications systems will replace wire based networks with fibre based optical systems. In order to achieve this goal, there are several technical challenges to be overcome to make this a practical reality. One of the most critical is the driver interface between the electronics and photonic modulator as this is crucial to the entire system performance.

With good progress in the implementation of high speed modulator drivers [1] a significant issue still to be resolved is that of the tolerance of the electronics to temperature variation. This is a particular issue in Silicon-Photonics systems due to high power density, where high temperature variation is inevitable. In differential Mach-Zehnder modulators (MZM), the effects of temperature are not a particular problem, however the overall performance of a transmitter is affected by electronic laser driver circuit. Therefore, the performance reduction of the laser driver due to temperature variation may jeopardise the overall performance of the system.

An example [2] is shown in the schematic diagram of an electro-optical transmitter with a MZM given in Figure 1. The transmitter consists of an Electronic laser driver (which is itself composed of a pre-driver and output buffer) and a Photonics integrated circuit (containing the modulator). The overall gain of the laser driver is accumulated in the stages of the predriver, and modulated optical signal of MZM is determined by voltage swing of output stage. A conventional solution independent of temperature uses dual-loop automatic power control [3], which uses a Photo-detector (PD) on the transmission side to provide feedback with the resulting additional costs on the optical device. $\mathrm{Li}$ and Chen [4] provided another

This work is partially funded by EPSRC Grant EP/L00044X/1

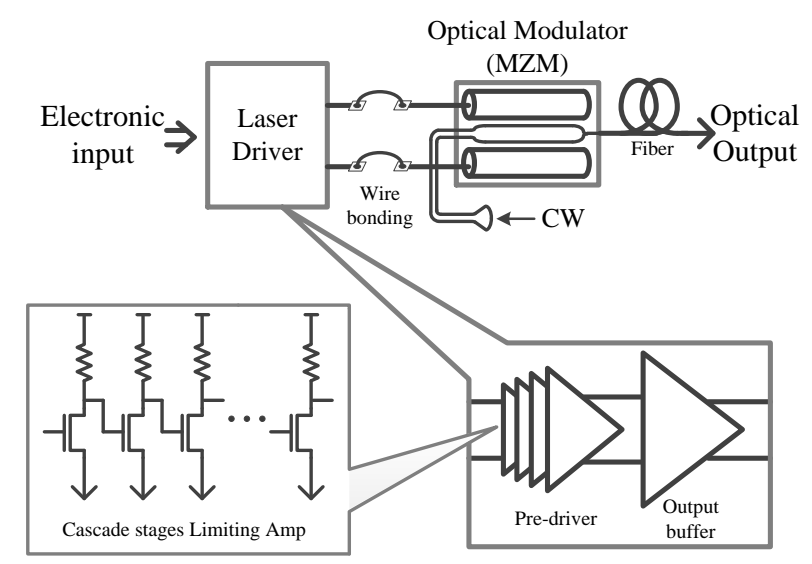

Fig. 1. An elec-optical modulation system with MZM

solution without additional optical devices, however this is a high cost and high power approach on the electronics side. This method required feedback from the electronic output to improve the overall signal quality. An integrated peek detector and thermistor control system off chip for tuning were used, makes it very complex, higher cost and not easy to integrate.

For wider applicability it is essential to have a scalable lowcost solution. In this work, the laser driver has been specifically designed to cope with temperature variation. The resulting overall performance is consistent across a wide temperature range.

\section{LASER DRIVER ANALYSIS AND IMPROVEMENT IN TEMPERATURE VARIATION}

\section{A. Effect of MOSFET in temperature variation}

Figure 2 presents the $g_{m}$ and $f_{t}$ characteristic of an NMOS transistor as the temperature increases. Assuming that such a device works as a common source amplifier with a passive resistance load, because the Gain passband $\approx g_{m} \cdot R_{L}$, the gain exhibits a $21.3 \%$ loss while the temperature increases from $27^{\circ} \mathrm{C}$ to $135^{\circ} \mathrm{C}$ as can be seen in Figure 2(a). Similarly, a huge reduction of peak $f_{T}$ from $63 \mathrm{GHz}\left(27^{\circ} \mathrm{C}\right)$ to $51 \mathrm{GHz}$ $\left(135^{\circ} \mathrm{C}\right)$ can be observed while increasing the temperature as shown in Figure 2(b). Therefore, as the temperature increases, both the bandwidth and gain of amplifiers base on MOSFETs will reduce.

\section{B. Output stage in Laser driver}

To assess the impact of temperature the comparions made between a current mode logic amplifier and a cascode push 


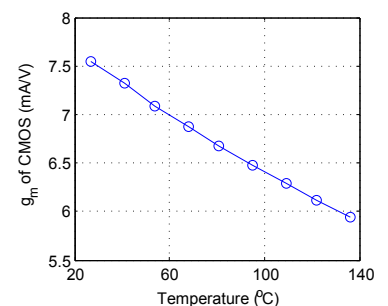

(a)

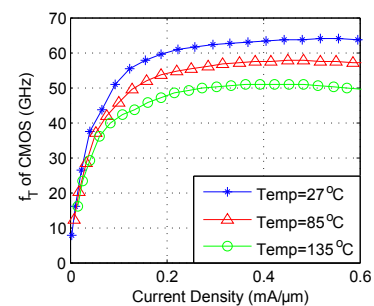

(b)
Fig. 2. (a) $g_{m} \&$ (b) $f_{t}$ of NMOS in temperature variation

pull amplifier (which can achieve $4 V_{p p}$ differential voltage swing). The current mode logic amplifier in Figure3(a) is driving a $50 \Omega$ termination with biasing. In Figure 3 b), there are two inputs in a cascode push pull with a $50 \Omega \mathrm{AC}$ termination. Both topologies were tested with same input voltage swing ( $V_{i n}=1 V p p$ ), and output swing (single rail) was observed to be higher than $2 V_{p p}$ at room temperature.
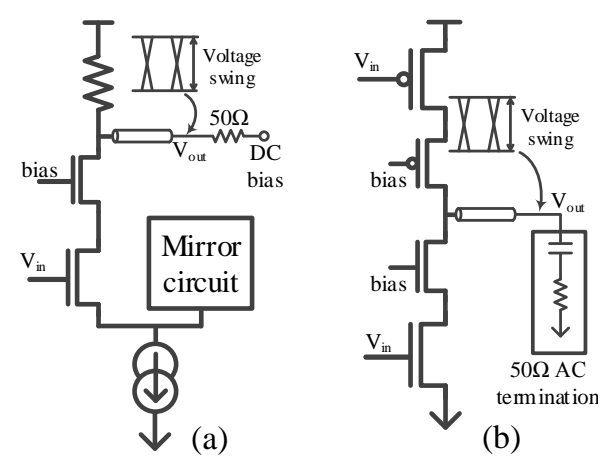

Fig. 3. Output stage of (a) current mode logic and (b) cascode push pull

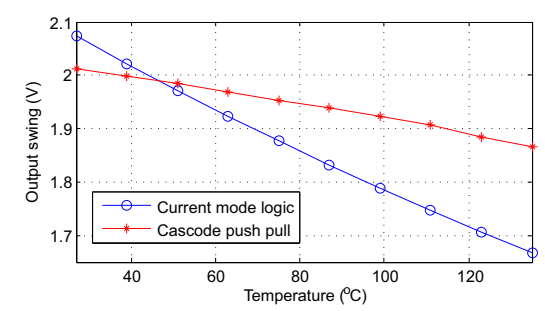

Fig. 4. Voltage swing of output stage

Figure 4 presents voltage swing of both topologies in wide temperature range. At high temperature, the gain loss of the current mode logic circuits results from a reduction of $g_{m}$ in the NMOS device. For example, at $135^{\circ} C$, the output voltage swing is reduced to $1.6 \mathrm{~V}$ ( $25 \%$ loss). In contrast, the cascode push pull architecture suggests much higher gain should be achieved but behaves as a buffer in this case. Most of the output voltage swing loss in a cascode push pull circuit is headroom loss, and in this case, it is only around $5 \%(150 \mathrm{mV})$. At high temperature, the bandwidth of both topologies is reduced. To compensate for this, a complex feedback system may be needed at the price of extra cost and more parasitics. Rather than these, the bandwidth loss in this single stage is acceptable for low cost applications. Overall, the cascode push pull circuit is a better solution taking these trade-offs into account.

\section{Limiting amplifier (pre-driver) in laser driver}

If it is assumed that each stage of the limiting amplifier has a gain of $A_{0}$ and a $-3 \mathrm{~dB}$ bandwidth at $\omega_{0}$, the transfer function of full limiting amplifier can be described by Eq. 1.

$$
H_{(s)}=\left(\frac{A_{0}}{1+\frac{s}{\omega_{0}}}\right)^{N}
$$

With an architecture consisting of $\mathrm{N}$ stages, the gain of the overall limiting amplifier is $A_{0}^{N}$ and the bandwidth is approximately[5], [6]:

$$
\omega_{-3 d B}=\omega_{0} \sqrt{\sqrt[N]{2}-1} \approx \omega_{0} \frac{0.9}{\sqrt{N}}
$$

Therefore, both the gain and bandwidth performance are affected by the architecture of the limiting amplifier as well as the laser driver. A limiting amplifier cell composed of three stage common-source amplifiers with 3rd order active feedback is shown in Figure 5. The bias current for M1 to M6 is set to $0.25 \mathrm{~mA} / \mu \mathrm{m}$. With a fixed passive resistor load, the frequency response of the whole circuit is shown in Figure 6 From $27^{\circ} \mathrm{C}$ to $135^{\circ} \mathrm{C}$, it can be observed that the pass-band gain loss is $3.6 \mathrm{~dB}$ and the bandwidth loss is $4 \mathrm{GHz}$. A general limiting amplifier comprises two or even three cascaded cells, as depicted in Figure 5.

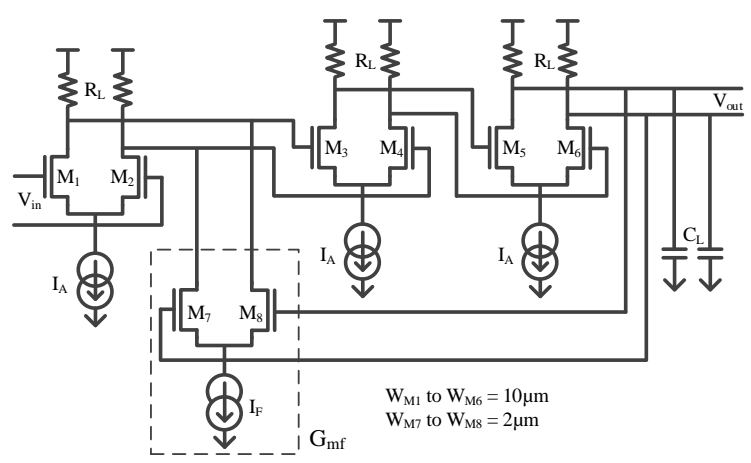

Fig. 5. A limiting amplifier cell with 3rd order active feedback

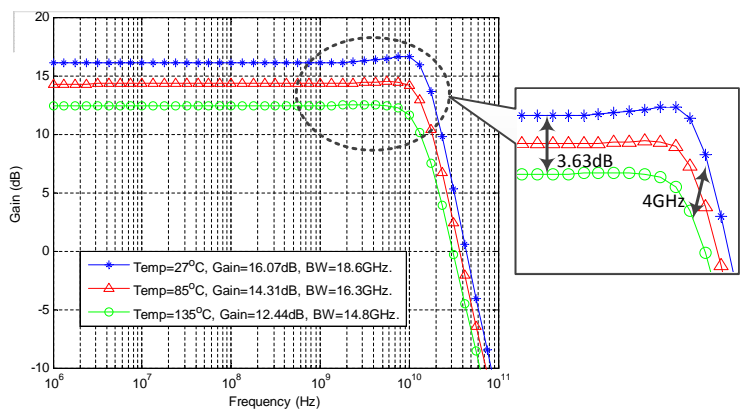

Fig. 6. AC response of the limiting amplifier cell

Figure 7 presents the topology of limiting amplifier cell with 3rd order active feedback, and its transfer function is given by,

$H(s)=\frac{A_{0}^{3}}{\left(1+\frac{s}{\omega_{0}}\right)^{3}+A_{0}^{2} \beta}=\frac{G_{m}^{3} \cdot R_{L}^{3}}{\left(1+s R_{L} C\right)^{3}+G_{m}^{2} G_{m f} R_{L}^{3}}$

As previously mentioned, the limiting amplifier loses both pass-band gain and bandwidth due to $G_{m}$ decreases at high 


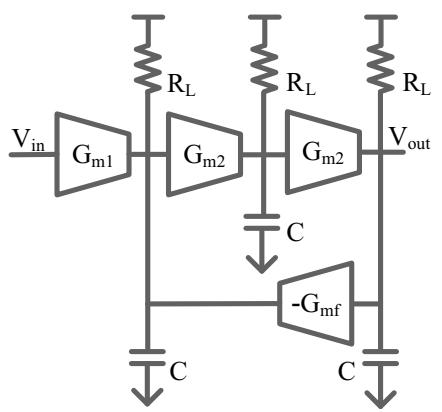

Fig. 7. 3rd order active feedback amplifier

temperature. Increasing $R_{L}$ balances the loss as well as maintaining the pass-band gain. Active feedback in this topology creates an extra pole in the denominator of the transfer function. Thus, increasing $G_{m f}$ could move the pole to higher frequency. In this design, the value of the load resistance and current source (which controls $G_{m f}$ ) were obtained empirically and are given below:

$$
\begin{aligned}
R_{L} & =300 \Omega+k_{1} \cdot\left(\text { temp }-27^{\circ} \mathrm{C}\right), \quad k_{1}=1 \Omega /{ }^{\circ} \mathrm{C} ; \\
I_{F} & =0.18 m A+k_{2} \cdot\left(\text { temp }-27^{\circ} \mathrm{C}\right), k_{2}=0.8 \mu \mathrm{A} /{ }^{\circ} \mathrm{C}
\end{aligned}
$$

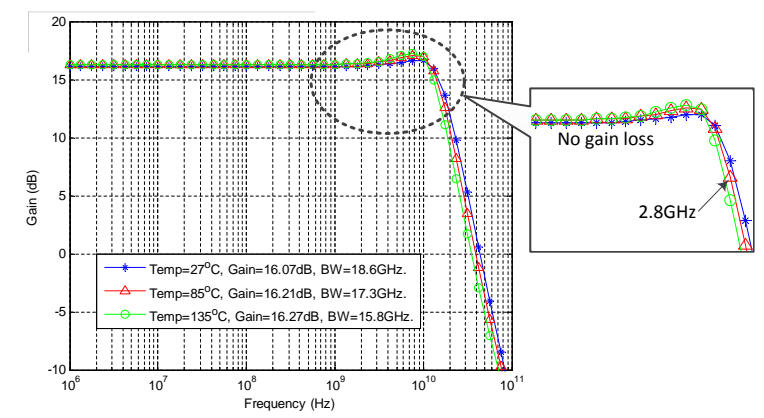

Fig. 8. AC response of improved limiting amplifier

Simulations of the frequency response shown in Figure 8 show the effects of temperature variation at $27^{\circ} \mathrm{C}, 85^{\circ} \mathrm{C}$, $135^{\circ} \mathrm{C}$. The gain and bandwidth of this limiting amplifier cell with adaptive active feedback and load are independent of temperature variation. The passband gain loss is less than $0.5 d B$ and the bandwidth loss is restrained within $2.8 \mathrm{GHz}$ in comparison with the previous results in Figure 6

\section{DESIGN EXAMPLE}

The following design example (Figure 9 ) is a fully differential laser driver for an MZM optical transmitter on IBM $130 \mathrm{~nm}$ CMOS process. With temperature independent limiting amplifier and cascode push pull output stage, the whole design produces similar performance on gain and bandwidth regardless of temperature. The temperature independent limiting amplifier regulated by temperature sensor and control circuit (Temp Ctrl), is acting as pre-driver for the output driver (Figure 10. Fully design is using DC supply at $3 \mathrm{~V} .1 / 2 V_{D D}$ is regulated by a integrated regulator.

In Figure 10, the output stage is an improved cascode push pull amplifier. In this design, a NMOS common source amplifier with a biased PMOS load behaves as a traditional

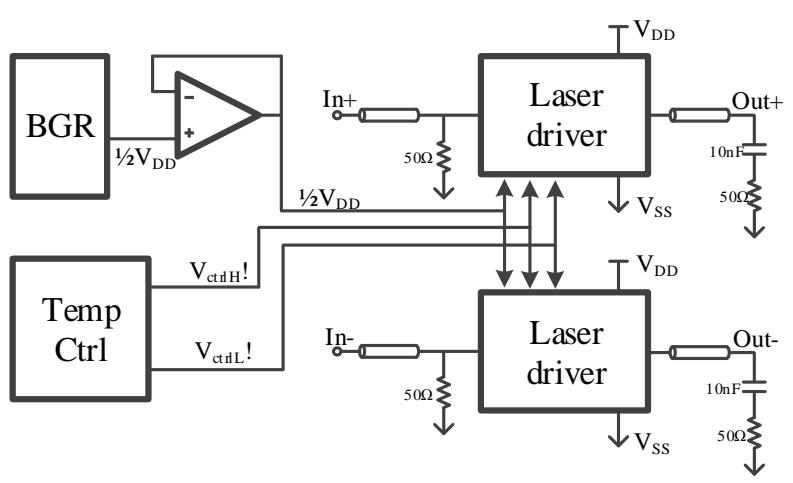

Fig. 9. Abstract diagram of laser driver with differential configuration

PMOS input (which would limit the bandwidth) is enhancing the bandwidth performance and reducing the input capacitance of cascode push pull output driver.

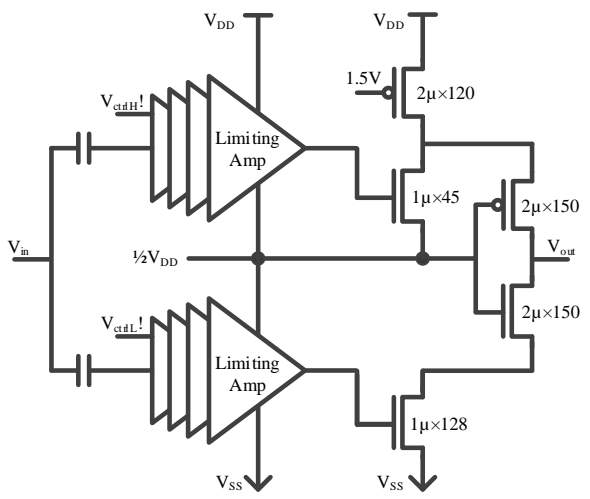

Fig. 10. Push pull cascode laser driver

The limiting amplifier cell is shown in Figure 11. It is an inductor-less amplifier with interleaving 3rd order active feedback, with similar circuit topology to [7]. The temperature independent function is achieved by using an adjustable resistive load and current shunt on $G_{m f}$. These adjustable components are composed of parallel MOSFETs with fixed bias and controlled bias. They share the control voltage (Vctrl!) from Temp Ctrl. While temperature increases from $27^{\circ} \mathrm{C}$ to $135^{\circ} \mathrm{C}, R_{L}$ and $I_{F}$ linearly increase similar to the trend in eq. $4 \& 5$.

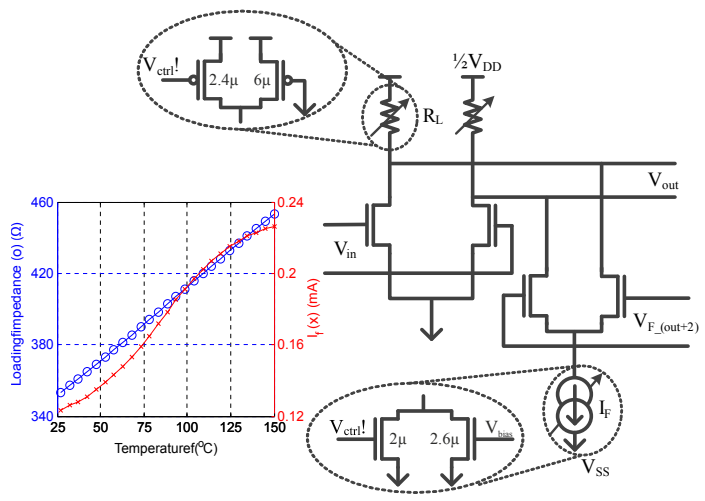

Fig. 11. Temperature independent limiting amplifier cell

The Pre-drivers, which are used for the laser drivers, are composed of 8 stages of limiting amplifier cells. To satisfy 


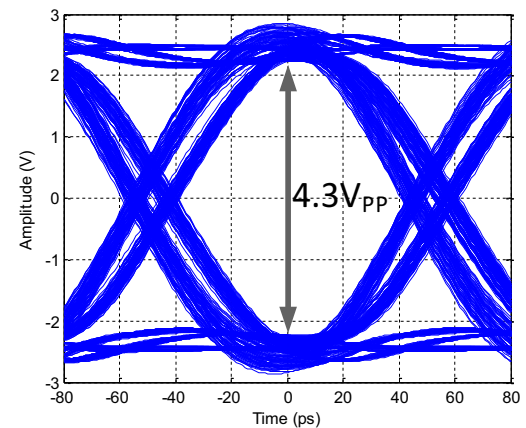

(a)

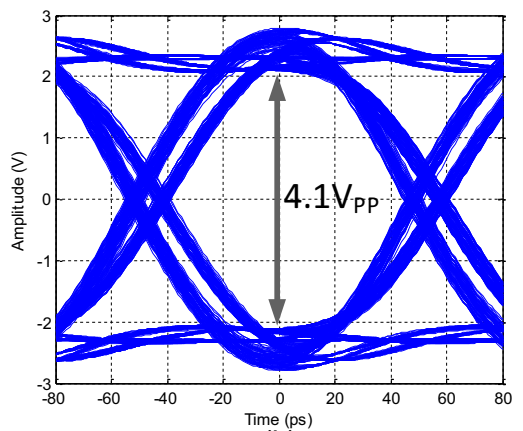

(b)

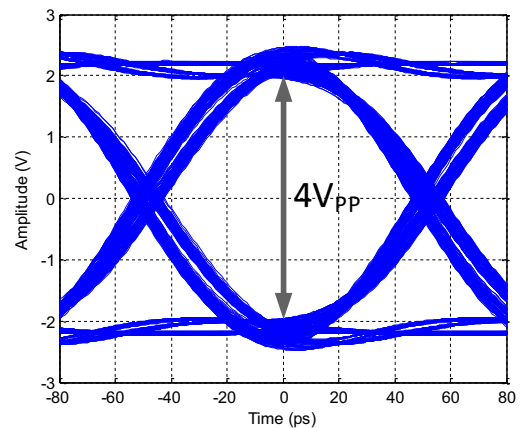

(c)

Fig. 12. Eye-diagrams of laser driver at speed of $12.5 \mathrm{~Gb} / \mathrm{s}$ with (a) $27^{\circ} \mathrm{C}$, (b) $85^{\circ} \mathrm{C}$ and (c) $135^{\circ} \mathrm{C}$.

the fan-out requirement of the output stage, the last 4 stages of limiting amplifier cells are a double fan-out design.

In order to simplify the system structure and save design area both adjustment components in limiting amplifier cells share the same control voltage. This voltage is generated by temperature sensor and control circuit in Figure 13

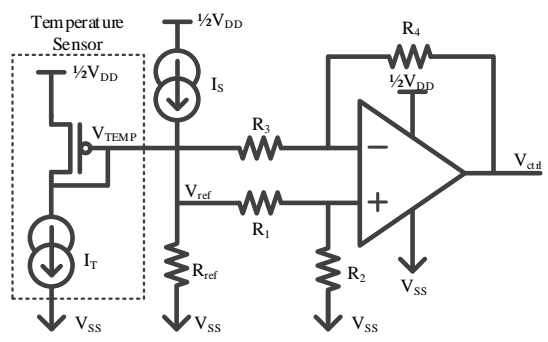

Fig. 13. Temperature sensor and limiting amplifier control

A temperature sensor is built with a temperature independent current source from a BGR and a self biasing PMOS. Because the reference voltage is proportional to the absolute temperature (PTVT) in PMOS (behavioral as same as a thermistor), Vtemp is increasing linearly along the increasing of temperature. Moreover, an OPAMP addition circuit is used to transfer Vtemp to a required control voltage (Vctrl!) for limiting amplifiers. In this case. this OPAMP exhibits high gain in wide temperature range and $V c t r l !$ is $250 \mathrm{mV}$ at $27^{\circ} \mathrm{C}$ and $650 \mathrm{mV}$ at $135^{\circ} \mathrm{C}$, providing a linear output voltage.

\section{RESUlts}

When running the post layout simulation with all parasitic effects ( $\mathrm{R}, \mathrm{C}$ and $\mathrm{L}$ ) and including temperature variation, a $12.5 \mathrm{~Gb} / \mathrm{s} 2^{31}-1$ pseudo-random binary sequence (PRBS) is applied to the driver, with a $100 \mathrm{mVpp}$ input. The output load is a MZM module with $50 \Omega$ AC termination. The power consumption of the whole system (include all DC circuits and temperature control system) is less than $377 \mathrm{~mW}$ (in all temperature conditions). The eye diagrams of the output signal are shown in Figure 12 . At $27^{\circ} \mathrm{C}, 85^{\circ} \mathrm{C}$ and $135^{\circ} \mathrm{C}$, the output voltage swing are all over $4 V_{P P}$ (differential) with an excellent signal quality. The full chip design area is less than $1 \mathrm{~mm}^{2}$ and the laser driver core area is less than $0.35 \mathrm{~mm}^{2}$. The layout view is shown in Figure 14

\section{CONCLUSION}

In this paper, a temperature variation independent laser driver for MZM has been proposed by exploiting adaptive lim-

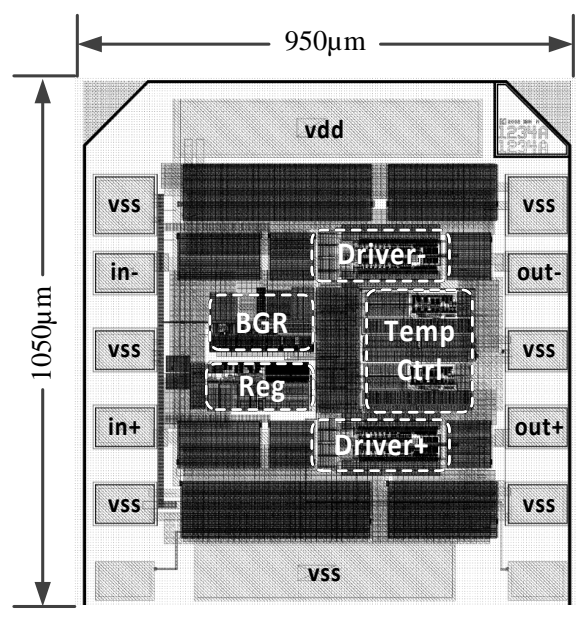

Fig. 14. Layout view of design example

iting amplifier and cascode push pull output stage. Moreover, this design has been implemented at relatively low cost compared to previous work. Post-layout simulation results with temperature information show that this laser driver maintains the performance in wide temperature conditions.

\section{REFERENCES}

[1] D.J. Thomson, F.Y. Gardes, Y. Hu, G. Mashanovich, M. Fournier, P. Grosse, J-M. Fedeli2 and G.T. Reed, "High contrast 40Gbit/s optical modulation in silicon", Optics Express, vol.19, iss.12, pp. 11507,11516, 2011.

[2] K. Li and P. Wilson, "An improved push-pull driver using $0.13 \mu \mathrm{m}$ CMOS," IEEE International Symposium on Circuits and Systems (ISCAS), pp.1958,1961, 19-23, 2013.

[3] D. Li and C. Tsai, "A 10Gb/s burst-mode/continuous-mode laser driver with current-mode extinction-ratio compensation circuit," Digest of Technical Papers in IEEE International Solid-State Circuits Conference 2006, pp.912,921, 6-9, 2006.

[4] D. Li; H. Chen, "10Gb/s $0.13 \mu m$ CMOS laser drivers with extinction ratio control using thermistors," Conference of Solid State Circuits, ESSCIRC 2007. 33rd European, pp.260,263, 2007.

[5] B. Razavi, "Design of Integrated Circuits for Optical Communications", 2nd ed, USA: Wiley. 2012.

[6] S. Galal, B. Razavi, "10-Gb/s limiting amplifier and laser/modulator driver in $0.18-\mu m$ CMOS technology," IEEE Journal of Solid-State Circuits, vol.38, no.12, pp.2138,2146, Dec. 2003.

[7] H. Huang, J. Chien and L. Lu, "A 10-Gb/s Inductorless CMOS Limiting Amplifier With Third-Order Interleaving Active Feedback," IEEE Journal of Solid-State Circuits, vol.42, no.5, pp.1111,1120, 2007. 